Fecha de recepción: diciembre 2019 Fecha de aceptación: febrero 2020 Versión final: marzo 2020

\section{Metadiseño y Transdiciplina, enfoque para la transformación social y ambiental}

Lorna Lares ${ }^{(1)}$

Resumen: En el escenario global actual, complejo y cambiante, el sentido de urgencia en alcanzar la carbononeutralidad, reducir los efectos de gases invernadero y los efectos del cambio climático, son parte de los desafíos que enfrentamos, no solo porque afecta la dependencia de los seres humanos de los combustibles fósiles sino también por la forma en que vivimos, como nos organizamos como sociedad y sus respectivas economías. Naomi Klein (2014), lo expresa claramente cuando dice: esto lo cambia todo. Para la ciencia, la tecnología y el diseño, innovación y sustentabilidad son fundamentales en el desarrollo de un nuevo modelo, aproximaciones y propuestas integrales, que aporten soluciones en los distintos niveles y dimensiones para poner en marcha las transformaciones estructurales que el planeta y la sociedad en su conjunto necesita.

Palabras clave: Sustentabilidad - Metadiseño - Transdisciplina - Transformación - Cambio climático.

[Resúmenes en inglés y portugués en las páginas 211-212]

(1) Lorna Lares es Master of Art in Design Futures. Goldsmiths, University of London, Inglaterra. Diseñadora Industrial, Universidad de Chile. Estudios en Letras, Universidad Central de Venezuela. Académica del Departamento de Diseño de la Facultad de Arquitectura y Urbanismo y Presidenta del Comité de Sustentabilidad, Universidad de Chile. Investigadora de la Red de Pobreza Energética Chile y responsable de la Línea de investigación: Bienestar, Eficiencia Energética y Contaminación Atmosférica. Investigadora asociada al programa transdiciplinario Energía, Agua y Sustentabilidad, Universidad de Chile. Especialista en metadiseño, sustentabilidad, eficiencia energética y economía circular. Vasta trayectoria en docencia del diseño, desarrollo profesional y gestión de diseño en instituciones de educación superior. 


\section{Introducción}

Es de conocimiento público que el planeta ha alcanzado, por primera vez en su historia, los niveles más altos de $\mathrm{CO}^{2}$ (415 partículas por millón de dióxido de carbono), aumentando casi en un 50\% desde 1990, y que dada la actual concentración y las continuas emisiones de gases de efecto invernadero, es probable que a fines de siglo el incremento de la temperatura mundial supere los 1,5 grados centígrados. Asociado a ello, los efectos son evidentes: olas de calor, erupciones volcánicas, aluviones, terremotos, incendios forestales, entre una larga lista de desastres. Por otro lado, sabemos que la energía es el factor que contribuye principalmente al cambio climático y representa alrededor del $60 \%$ de las emisiones mundiales de efecto invernadero, y que las ciudades ocupan solo el $3 \%$ de la tierra, pero representan entre el $60 \%$ y el $80 \%$ del consumo de energía.

En este contexto, la evidencia científica es irrefutable, ha demostrado con creces el impacto de la actividad humana sobre el planeta, desencadenando el progresivo aumento de la población mundial y su concentración, los cambios de uso de suelo, los procesos de urbanización con altas emisiones de contaminantes nocivos para la salud, y el sobreconsumo enmarcado en un modelo de desarrollo basado en una visión antropocéntrica, extractivista y depredador.

Desde una perspectiva del diseño, como área proyectual -además de su práctica profesional- se debe asumir la responsabilidad del ser y el hacer del diseño en los impactos que generan en las distintas dimensiones y escalas (desde lo local y su consecuente implicancias en la escala global), respondiendo a lo emergente de manera creativa y consciente en los efectos del uso de recursos, impactos asociados en cada etapa del proceso, en el planeta, en la sociedad, estilos de vida y las personas.

Nutrir al diseño, como transformador de estos desafíos, requiere de una reorientación con nuevas aproximaciones y metodologías, que contemple una visión más holística, ecomimética y transdisciplinar; se requiere de un nuevo marco conceptual y referencial que nos permita re-definir o re-significar el diseño. Además de crear las infraestructuras sociales y técnicas donde tengan lugar nuevas formas de colaboración y co-creación. Para ello, se requiere del metadiseño, como enfoque y como herramienta.

El sistema económico imperante y los problemas medioambientales han puesto históricamente (desde la revolución industrial) a los profesionales proyectuales (en especial aquellos con enfoques funcionales y racionales), en un dilema ético, siendo llamados, en la mayoría de los casos, como especialistas para promover el consumo y fomentar el crecimiento económico. Ello, en parte, debido a su formación especializada (delimitada por su propio lenguaje y técnica), orientada a resolver "problemas" desde las fronteras de su propia disciplina y desde su aproximación a la forma y traducción en productos/servicios para satisfacer las "necesidades" de los clientes, usuarios o personas, dependiendo del área económica-social con que se relaciona. Sin embargo, los desafíos globales urgentes obligan a repensar la organización disciplinar para articular el conocimiento con la crisis medioambiental, los efectos del cambio climático, las trasformaciones hacia un nuevo habitar y con-vivir, donde la noción tradicional del diseño necesita extenderse más allá de sus propias fronteras disciplinares. En consecuencia, se necesita un nuevo tipo de profesional, metadiseñadores capaces de trabajar en un alto nivel organizacional, de manera holística, 
transdisciplinar, ética y eco-mimética, pues a pesar de todo el aporte y esfuerzo del Diseño Sustentable (Design Council. UK, 1980), del Ecodiseño (1990), entre muchas otras aproximaciones "verdes", el agotamiento de los recursos, la sobreproducción, la sobreabundancia de desechos y la contaminación ambiental continúan siendo un problema y un desafío para las futuras prácticas del diseño en pos de un desarrollo sustentable y de cara a los desafíos globales urgentes: energía, agua, biodiversidad, migración, envejecimiento, igualdad de género, entre otros.

\section{La necesidad de una educación hacia el Desarrollo Sustentable}

El tiempo y la tecnología cambia rápidamente surgiendo con ello nuevos requerimientos que condicionan los dominios de especialidad de los profesionales y la manera como estos trabajan, así como el nivel de impacto en el mundo en que vivimos. Sin embargo, el progreso en educación para el desarrollo sustentable ha sido muy lento, un ejemplo de ello es que desde que las Naciones Unidas se declaró el Decenio de la Educación con miras al Desarrollo Sustentable (2005-2014), teniendo como objetivo general "integrar los principios, valores y prácticas del desarrollo sostenible en todas las facetas de la educación y el aprendizaje" (Unesco, p. 6) ${ }^{1}$ el avance que hemos tenido en el desarrollo de soluciones y propuestas educativas en este contextos han sido muy escasas, sobre todo en América Latina y especialmente en Chile.

El rol de las instituciones de educación superior (IES) es relevante y decisivo en la transformación cultural, social y económica de una sociedad sustentable, pues constituyen una plataforma para el desarrollo y aplicación del conocimiento -deseablemente vinculante con las políticas públicas-; la formación de profesionales éticamente responsables con el medioambiente; el ordenamiento territorial, urbanización y desarrollo sustentable de las ciudades; valoración e integración de las organizaciones públicas y privadas, comunidades y pueblos originarios en la configuración ecosocial.

Aun cuando, en Chile, existen algunas IES con avances en materia de extensión o vinculación con el medio, gestión y administración de campus, investigación y docencia (integrando competencias de sustentabilidad en sus mallas curriculares, incorporando contenidos en distintos programas y asignaturas, entre otras), no se visualiza un avance significativo en temas de gobernanza e institucionalidad que centre especial atención en un modelo educativo que permita el problema ser parte de un ecosistema. Es necesario una estructura donde las relaciones y conversaciones disciplinares se crucen, entrelacen y se asocien, abordando los desafíos desde una manera más integrada.

La sustentabilidad es abordada aún de forma compartimentada y no como motor de desarrollo o principio fundamental para alcanzar una educación con valores, actitudes y habilidades que mejore la calidad de vida de las actuales y futuras generaciones, el actual nivel de sobreconsumo, los niveles de equidad, los problemas medioambientales, la sobreexplotación de recursos y las prácticas productivas insostenibles, para generar nuevos patrones de desarrollo, costumbres y estilos de vida más saludables y socio-ambientalmente sustentables. Lo anterior pone en cuestionamiento, al menos, si la educación actual está siendo el 
problema o la solución. Se hace necesario reorientar la educación hacia la sustentabilidad que propicie una ética distinta al antropocentrismo y entregue el espacio para nuevas formas de relaciones. Asumiendo que, en estas relaciones, somos partes del sistema, pero también que cada uno depende de otro en un sistema relacional, donde se puede contribuir positivamente o negativamente en el bienestar humano y del medioambiente.

En consecuencia, se requiere una mirada holística, inclusiva y un trabajo transdisciplinar, que involucre a diversos actores, generar nuevas formas de diseño que incidan en la toma de decisiones a favor de soluciones integrales y adaptativas a temas tan urgentes como la reducción de gases de efecto invernadero y la inseguridad alimentaria (disponibilidad y acceso). Según el Fondo Monetario Internacional y el Banco Mundial (2011), 795 millones de personas en el mundo pasan hambre y 702 millones de personas viven en extrema pobreza (1,9 dólar al día), ello representa entre un $11,3 \%$ y un $10 \%$ aproximadamente de la población mundial, estimada en más de 7000 millones al 2011. Del mismo modo, en el área de los recursos naturales, el acceso a agua potable (disponibilidad y acceso), se estima que para el 2050 nazcan 3000 millones de personas en el mundo y se proyecta que será en países donde actualmente ya se está experimentando déficit de agua (China, India y México). Chile, si bien es un país donde gran parte de su actividad económica se basa en la agricultura, y es uno de los países con las mayores reservas de agua, presenta un problema adicional en el acceso equitativo a los servicios energéticos de alta calidad, para cubrir sus necesidades fundamentales y básicas, que permitan sostener el desarrollo humano y económico de sus habitantes. Dentro de los servicios energéticos fundamentales (que implican impacto directo en la salud humana), se encuentra la cocción y conservación de alimentos y el acceso al agua. La posibilidad de refrigeración y cocción de los alimentos permite a los hogares una mejor administración de sus recursos y aumentan la probabilidad de acceso a una dieta nutricionalmente variada y suficiente (RedPE, 2019)2 2 Del mismo modo, el acceso al agua caliente sanitaria se transforma en un indicador de pobreza energética en Chile, debido a que 10,2\% de los hogares no cuenta con este servicio (CASEN, 2017) ${ }^{3}$.

Estamos ad portas de cumplir casi cuatro décadas desde que Brundtland Commission publicó Our Commun Future, donde se estableció la definición de desarrollo sustentable, como "...el desarrollo que satisface las necesidades del presente sin comprometer la capacidad de las generaciones futuras para satisfacer sus propias necesidades" (WCED, 1987: 24) ${ }^{4} \mathrm{y}$ pareciera que esas futuras generaciones aún están muy distantes de satisfacer sus necesidades y las de la naturaleza. En consecuencia, las generaciones actuales, los estudiantes de hoy y profesionales de mañana, mantiene un sistema, que si bien está experimentando modificaciones en el modelo educativo, no son lo suficientemente profundos como para hacer las transformaciones que las sociedades necesitan para adaptarse a los cambios. Cambios que inevitablemente sucederán. Cambios acelerados y profundos en el medioambiente, pero pareciera que aún estamos esperando que otros tomen las decisiones políticas, sociales y económicas para dar un giro definitivo a nuestra educación y a la formación de una nueva generación de profesionales proyectistas, entre ellos los diseñadores, quienes sin duda están impactando y generando parte de las consecuencias con su quehacer al configurar el entorno material -desconfigurando la estructura del entorno natural e inmaterial-, alimentando un estilo de vida basado en el consumo -sobreproducción y extracción 
exacerbada- para mantener un el modelo económico imperante. Si la educación es parte de la solución, sino la solución, entonces se requiere de un profundo cambio y una amplia visión de futuro, que va más allá de las especializaciones estanco, de disciplinas proyectuales con incidencia compartimentada.

\section{Metadiseño como disciplina, estructura flexible y auspiciosa para las transformaciones}

El biólogo Humberto Maturana, una década más tarde de la definición de desarrollo sustentable, en su ensayo sobre Metadiseño expone que

Vivimos en una cultura centrada y guiada por relaciones de dominación y de sometimiento, en la desconfianza y el control, la deshonestidad, el comercio y la codicia, la apropiación y la mutua manipulación... a menos que nuestro emocionar cambie nuestras vidas seguirán un camino que nos llevara a lo mismo: guerra, codicia, desconfianza, deshonestidad y abuso de otros y de la naturaleza. De hecho, permaneceremos igual (Maturana, 1997).

Desde entonces la noción de poner el énfasis en temas tan relevantes y esenciales como las emociones y la conciencia humana, la responsabilidad y el quehacer profesional, en la educación y el desarrollo de una cultura más responsable con el medioambiente. Sin embargo, seguimos manteniendo las mismas prácticas pedagógicas en las universidades. Si bien algunas ofrecen programas con tópicos relativos a la sustentabilidad, otras solo han incorporado, esporádicamente, temáticas sobre cambio climático, conservación de la energía, pobreza energética, diversidad cultural y agotamiento de los recursos, en algunos de cursos. El foco pareciera estar en el reciclaje y la reutilización de materiales, como es el caso de la Ley Marco para la Gestión de Residuos, la Responsabilidad Extendida del Productor y Fomento al Reciclaje. Ley n²0.920, o más conocida por sus siglas REP, en Chile. Esta busca el reciclaje de un grupo de materiales, tales como: aceites lubricantes, aparatos eléctricos y electrónicos, baterías, pilas, envases y embalajes, neumáticos, y cuyo objetivo general es

Establecer un marco jurídico para la gestión de residuos y, en particular, instaurar la responsabilidad extendida del productor, todo ello con la finalidad de incentivar la prevención en la generación de residuos y fomentar su reutilización, reciclaje y otro tipo de valorización, protegiendo así la salud humana y el medio ambiente (MMA, 2016: 1) .

Estas acciones sin duda son importantes, pero ello no aborda o resuelve el origen del problema. Se plantea como un agente mitigador que resuelve el desecho, pero no evita su generación o incorporación al ciclo de vida. Más aún, el producto resultante, muchas veces consume más recursos y energía en su transformación y/o se convierte en un objeto de consumo mayor, que pareciera validar la generación del residuo. 
McDonaugh y Braungart (2002) sostienen que utilizar el término "reciclar" para describir el sistema de reutilización es poco honesto, ya que en cada uso posterior se genera un material de grado inferior hasta que finalmente nos quedamos con desechos inservibles que solo pueden ser incinerados o acumulados en vertederos. En contraposición plantean la necesidad de eliminar el concepto de desecho y sustituirlo por el diseño de sistemas y productos que al final de su vida útil den sustento a algo nuevo, es decir el residuo como un recurso diseñado y no centrarse en la compleja tarea de reciclar o reutilizar materiales que no han sido pensados para tal fin. Como profesionales y facilitadores responsables, así como ciudadanos y diseñadores globales, tenemos el deber de asegurar prácticas docentes y laborales enmarcadas en criterios sustentables y gatillar los cambios culturales necesarios para asegurar la transformación y adaptación hacia un mundo más sustentable, sin comprometer hoy las necesidades de las generaciones futuras.

El diseño ha tenido siempre un papel preponderante en este tema, las ideas de Víctor Papanek (1972), un adelantado para su tiempo, plasmadas en su libro Diseñar para un mundo real: ecología humana y cambio social, ya proponía un enfoque sustentable al denunciar la obsolescencia del sistema y considerar que los diseñadores deberían centrar su quehacer en favor del bien común y no solo en pro de las empresas, el consumo y el bienestar financiero, pensaba que los diseñadores estaban implicados en toda contaminación de alguna manera y que a los diseñadores solo les interesaba jugar un papel pequeño y raramente atender las necesidades reales. Sin embargo, creía que los diseñadores tenían las habilidades y actitudes suficientes, así como un rol decisivo en la configuración de un mundo más sustentable pues tenían la sabiduría para anticipar las consecuencias medioambientales, ecológicas, económicas y políticas de las intervenciones de diseño. Asimismo, otro visionario, el científico, inventor y activista medioambiental Buckminster Fuller ha servido de inspiración a muchos diseñadores a través de su máxima de hacer más con menos y con su idea de integrar los sistemas naturales a lo creado por el hombre, así como la defensa por la distribución equitativa de las riquezas y la igualdad social, pero por sobre todo su obra e ideas presurosas del movimiento contemporáneo en pos del desarrollo sustentable. Todo ello nos lleva a cuestionarnos si en un mundo en constante cambio, acelerado, con cada vez más y mayor tecnología, con una sociedad que persigue un diseño y producción material en pos del consumo y crecimiento económico, cuando ya desde 1970, distintos profesionales y autores, como Papanek llamaba a los diseñadores (en el amplio sentido de la definición) a abandonar el concepto "design for profit" o abandonar la sola idea de diseñar para el beneficio de las empresas - industria - mercado; cuando todos los tratados y cumbres en pos del cambio climático y un desarrollo sustentable, no han permeado las prácticas de las disciplinas del diseño, haciendo evidente la necesidad de redefinir las practicas actuales (con valores éticos y responsables) y sobrepasar las fronteras de estas para el beneficio de una amplia sociedad, entonces la pregunta que surge es:

¿Debemos mantener nuestro ámbito de acción en la forma y en la configuración de productos/ servicios, como lo hemos mantenido hasta hoy?,

Aún más ¿Debemos mantener las fronteras?

Con las capacidades y habilidades que ya en su tiempo Buckminster Fuller atribuía a los diseñadores, debemos ser capaces de redefinir nuestro quehacer y abordar problemáticas que van más allá del objeto e incidir más directamente en la relación economía nacional y 
problemas de pobreza o desigualdad, teniendo la facultad, responsabilidad ética y social, para crear estilos de vida y en consecuencia un mundo más sustentable. Toda vez que los diseñadores toman diariamente decisiones que implican el uso de recursos renovables y no renovables, el uso de productos y definición de estilos de vida, decisiones sobre comunicación y maneras de habitar que inciden directamente en hábitos y costumbres, sobre explotación de recursos y preservación de especies. Hay que asumir que el diseño cambió. Todo lo anterior exige una formación que permita ir más allá de las fronteras actuales de la disciplina, hacia el Metadiseño.

\section{Metadiseño como un modo de re-definición hacia el desarrollo sustentable}

El término Metadiseño ha sido usado desde 1980 especialmente en el área de la tecnología de la información con referencia al arte, teoría cultural y prácticas de diseño. No obstante, no es ni una disciplina establecida ni una teoría exacta, la idea de metadiseño ha producido diferentes entendimientos y aplicaciones, así como metodologías y objetivos en distintos campos de aplicación, todo ello desarrollado acorde con cada interés particular, según el contexto y autor. Si analizamos etimológicamente, la palabra "meta" significa en griego: después, posterior, más allá de, cambio de lugar, orden o naturaleza. Para el diseño, visto como una trasformación, un alto orden u orden diferente, o un reajuste de algo (Etymology, 2006). Youngblood (1984), lo define como "la creación del contexto en lugar de la creación de contenido" (citado por Giaccardi, 2003:75).

Asimismo Giaccardi (2003) en su tesis "Principles of Metadesign: Processes and Levels of Co-Creation in the New Design Space" lo define como

(...) la expresión de preocupación e intención que nos puede llevar de vuelta a un campo interconectado de significado y conceptos, recomponiendo el tejido de una cultura de diseño coherente y como respuesta original a los cambios que se produce en/o condiciones materiales y existenciales (Giaccardi, 2003: 69).

Lo describe como una cultura de diseño que emerge desde el contexto de las actuales teorías de diseño y como un intento para responder efectivamente a la nueva condición material y de existencia del presente mundo. Argumenta que es un marco conceptual que crea y define infraestructura social y técnica en el cual nuevas formas de diseño colaborativo pueden tener lugar. Por otro lado, para el arquitecto, ingeniero y diseñador Ezio Manzini, metadiseño es "el diseño de un conjunto de herramientas, metodologías y formas para respaldar a los diseñadores en su quehacer dentro de una variedad de procesos de diseño" (citado en Utopías, 2007).

Ambas perspectivas, otorgan un grado amplio campo de acción y de posibilidades. Por un lado, la idea de una nueva filosofía del diseño, una cultura, como lo propone Giaccardi, para producir nuevas relaciones y transformaciones, y por otro lado, complementario a ello, una herramienta o metodología que se adapta para responder - proponer - crear so- 
lución a los distintos desafíos a los que deben dar respuesta los diseñadores. Es decir, una estructura organizacional flexible, adaptable y consensual.

En las ciencias biológicas se define metadiseño como la naturaleza de coexistencia de los organismos vivos junto a su potencial para generar nuevas dimensiones relacionales, ofreciendo diversas posibilidades para una coexistencia social y no-social y para crear una nueva realidad en el curso de la historia humana (Maturana, 1997: 11). Asimismo, Maturana en su famoso artículo "Metadiseño" define este concepto como un acto creativo responsable el cual tiene el potencial para describir cómo los organismos vivos co-existen, favoreciendo la base de la existencia humana, vinculando el concepto en cierto modo al concepto de autopoiesis. Una visión ecosistémica vinculada a la auto-organización. Coincidiendo con la idea de "Un modo de diseño que se diseña a sí mismo como un todo para redefinir y sinergizar las infraestructuras culturales $y$ sociales de manera colaborativa y adaptativa" (Fuad-Luke, 2009: 22). Ambas concepciones se centran en la estructura organizacional, donde el centro no es el resultado objetual o de servicio, sino las relaciones que se producen entre las variables - cambiantes- del sistema.

Por otro lado, John Wood (2007) plantea un cambio de paradigma con base en el metadiseño, visto como una aproximación eco-mimética centrada en el lenguaje. Argumentando que el metadiseño podría emerger desde una continua visión consensual de lo que está pasando, lo que no podría lograrse unilateralmente, y una manera diferente a lo que normalmente conocemos como diseño.

Probablemente, estas definiciones, concepciones o ideas de metadiseño podrían generar mayor confusión conceptual, de lo que produce con el concepto de diseño. Sin embargo, si tomamos la idea de metadiseño como un modo para la redefinición de los actuales modelos, sean ellos los propios modelos de diseño, los modelos de consumo y su consecuente responsabilidad medioambiental, podría ser un camino para alcanzar las transformaciones necesarias para la adaptación, que nos permitiría un desarrollo sustentable. Argumentado desde la base que es un modo de diseño más holístico, consensual, integrado, ético y eco-mimético, que trabaja en un alto nivel de organización, en orden a sinergizar los factores que más contribuyen al agotamiento de los recursos, la sobreproducción, la alienación y los residuos (Wood, 2007) y donde los diseñadores necesitan trabajar de manera multidisciplinaria y transdiciplinaria (en colaboración con otros diseñadores, otros especialistas, comunidades y personas, en distintos territorios y ciudades) lo que permitiría generar una nueva cultura ecosocial, ética y responsable con mayor conciencia en el impacto del resultado de su quehacer teórico y práctico, en un mundo cada vez más complejo.

La idea de un alto nivel de diseño donde se introduzcan nuevas relaciones de competencias, valores y habilidades para enfrentar este mundo complejo, generaría nuevos contextos y estilos de vidas más simbióticos y eco-miméticos, que nos llevarían a superar los límites que hasta hoy no hemos podido dar por superados con la idea de sustentabilidad por sí sola (Ver Figura 1). 


\section{Education}

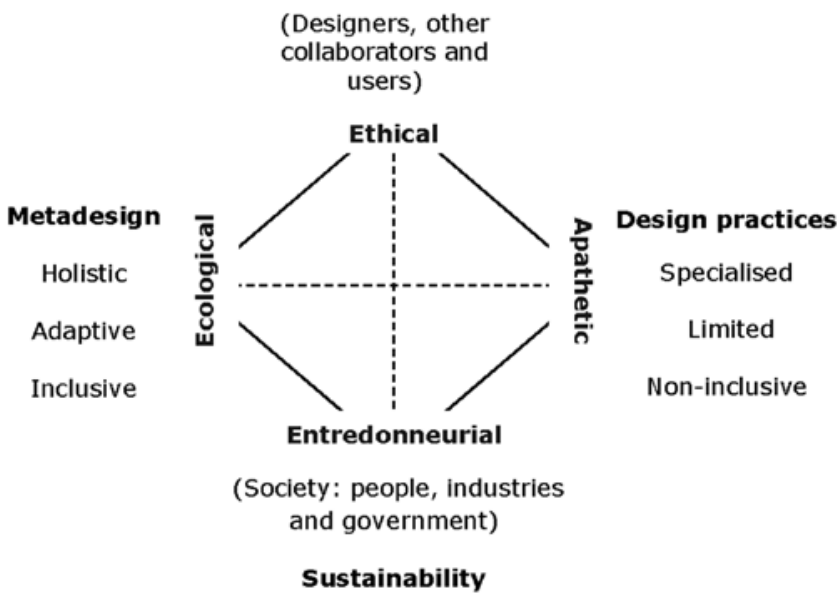

Figura 1. Modelo Tetrahedronal (Fuente: Elaboración propia).

El diseño y los diseñadores han entregado, sin duda, una importante contribución a los temas ecológicos, preservación de los recursos naturales y biodiversidad, equidad social, acceso a educación y salud, así como a la superación de la pobreza. Sin embargo, la evidencia demuestra que no es suficiente y que su impacto no ha sido muy significativo, quizás se deba al bajo nivel de intervención y al rol que cumplen en la toma de decisiones en la escala organizacional; o quizás, como ya planteaba Papanek (1984) “(...) Hay pocas profesiones más dañinas que el diseño industrial [además de otras especialidades del diseño]. Hoy el diseño industrial ha permitido la producción en cadena del asesinato [aún más] los diseñadores se han convertido en una especie peligrosa" (Papanek 1984: ix). reflexionando sobre los débiles acuerdos gubernamentales en las recientes cumbres sobre el Medio Ambiente y el Desarrollo Sostenible (Río+20, Junio de 2012) y el COP21 de París (diciembre de 2015) (citado por Escobar, 2016: 25).

Estas visiones, en el contexto actual, sirven de fundamento para concluir que algo no está funcionando. El Diseño cambió. Como afirma Manzini (2015) "la industria tal como la conocimos es un paradigma acabado, producir objetos es hoy una tarea del pasado y ante el repliegue de los objetos, solo quedan las personas. Así, su perspectiva pone al sujeto -y no al objeto- en el centro del debate" pues los objetos/servicios que dan forma y moldean los estilos de vida del mundo, son parte de los grandes problemas: contaminación ambiental, escasez hídrica, sobreconsumo, crisis social y climática.

Todo lo anterior, se deba en parte, a que los diseñadores están siendo formados para ser especialistas, educados bajo un sistema que incorpora niveles de competencias profesionali- 
zantes que incentivan las necesidades de la actual forma dominante de la modernidad capitalista. Una vía para superar esta situación es crear sistemas de gobernanzas, metodologías, herramientas y redes para integrar distintos niveles del conocimiento y distintos actores. En base a estructuras colaborativas, adaptativas y resilientes, en favor de volver a una dimensión cultural del diseño, más holística, reflexiva, más humana y consensual, que permita pensar otras maneras de desarrollo, más allá de la visión -hacer y ser- de las disciplinas. Un cambio cultural donde lo humano este en relación de semejanza con lo no humano.

En este sentido, la idea del metadiseño, como una cultura de diseño, puede ser una poderosa metodología, herramienta y práctica para trabajar en un alto nivel de colaboración y participación, que responda a las demandas actuales y a nuevos escenarios complejos, así como a las condiciones existenciales del mundo actual, ya que éste no solo trabaja en un nivel estratégico u organizacional sino también a un nivel emocional, tal como dice Maturana (1997) "si queremos un cambio necesitamos un cambio cultural en el dominio de la existencia humana” (Maturana, 1997:13), para alcanzar un mundo más relacional y natural. Una exploración en el ámbito del metadiseño es una oportunidad para abordar y potenciar las capacidades humanas y profesionales, además una oportunidad de aplicar el concepto de co-creación ${ }^{7}$ junto al concepto de desarrollo sustentable y la creación de nuevos escenarios inclusivos con resultados que no somos capaces de visualizar ahora, como algunas esferas impredecibles y dinámicas colectivas en estados no planeados producto del trabajo con la incertidumbre.

\section{Transdisciplina hacia el otro -con el otro- diseño}

Piaget (1976), el primero en introducir el término transdisciplina en la ciencia, postula que cada una de las ciencias es parte del sistema de las ciencias, donde se generan procesos de interacción e interdependencia que pueden conducir a la integración y a una serie de modelos relacionales que incluyan tanto las diferencias como las semejanzas de las distintas aportaciones disciplinarias (Luengo, 2012: 14).

La división disciplinar, nos dice Luengo (2012), ha sido y es productiva, pero la sola disciplina aislada no lo es. Las disciplinas requieren del cruce, de la intersección, del reconocimiento de las encrucijadas del conocimiento, y de trabajar en los límites de lo que consideran su objeto de estudio. Por ello, el conocimiento invita tanto al trabajo disciplinar como a la inter y transdisciplina, no sólo como necesidad teórica sino también como exigencia práctica. Al fragmentar constantemente los problemas, para que sean atendidos por áreas de especialización, ignoramos el contexto más amplio que da a las cosas su sentido y significado en el conjunto. De hecho, este espíritu de fragmentación está permeando no sólo la ciencia y la tecnología sino la visión general de la realidad que tenemos como individuos. Solemos centrarnos y circunscribirnos a problemas parciales y concretos, siendo que estos están relacionados con un entorno en movimiento más amplio, que recibe los efectos positivos y negativos de las respuestas que se ofrecen a los problemas tratados aisladamente (Luengo, 2012: 9). Tal relación fundamenta lo expuesto en el apartado anterior y refuerza la idea de una nueva cultura del diseño como el metadiseño, asociado a la transdisciplina. 
En ese sentido, el conocimiento específico de la disciplina es necesario y complementario porque enriquece y nutre la transdisciplina. No es que debamos dejar de enseñar la disciplina, el desafío esta en qué y cómo aportamos en la orquesta transdisciplinar, ya que, como plantea Piaget (1972), la transdisciplina está relacionada con el cruce de fronteras disciplinares y de otro tipo de saberes en la construcción del conocimiento. Sin embargo, no se detiene en las "interacciones y reciprocidades entre conocimientos e investigaciones especializadas, sino que sitúa esas relaciones entre disciplinas al interior de un sistema sin fronteras entre las disciplinas" (citado por Luengo, 2012: 11).

\section{Metadiseño y sus desafíos en la docencia universitaria}

(...) El diseño está evolucionando desde su posición de relativa insignificancia dentro de los negocios ( $y$ el campo más amplio de la naturaleza) para convertirse en el mayor proyecto de todos [...] El cambio masivo no es sobre el mundo del diseño; es sobre el diseño del mundo (Mau en Escobar, 2016).

Desde esta estructura conceptual, se propone como ejemplo de análisis, un caso concreto que se está desarrollando en pos de una redefinición de un modelo; en este caso, el modelo actual de enseñanza del diseño industrial e ingeniería eléctrica. Enmarcado en el interés nacional actual por impulsar fuertemente la innovación en el área de la energía, diversificando y enriqueciendo la creación de nuevos sistemas de generación, distribución y acceso; potenciando la creatividad nacional y fortaleciendo de soluciones locales a problemáticas globales. En este contexto, la iniciativa de la Corporación de Fomento de la Producción (CORFO) está invirtiendo considerables recursos en fomentar estas competencias en las universidades que imparten programas de ingeniería del país, bajo el programa "Una Nueva Ingeniería para el 2030".

Apoyado por este Programa, en la Universidad de Chile, los recursos se han enfocado no sólo en potenciar la formación entre distintas ingenierías, sino también se reconoce la vital importancia de generar lazos con otras disciplinas, tales como el diseño. Particularmente, mediante la iniciativa Beauchef Proyecta ${ }^{9}$, se está motivando la formación de profesionales líderes, capaces de aportar soluciones integrales desde una perspectiva transdisciplinaria, a través de la generación de espacios de vinculación y creación conjunta entre diseño e ingeniería, basada fuertemente en el desarrollo de pensamiento holístico, sistémico e integral, con una cultura enfocada en el proyecto.

Desde la academia, investigadores, profesores y estudiantes definieron como espacio articulador la docencia de pregrado, asociando cursos semestrales de los programas de estudio de diseño industrial e ingeniería, en un formato laboratorio-taller que permite la co-creación entre ambas disciplinas. Los cursos en cuestión corresponden a Proyecto III y IV (tercer año) de la carrera de Diseño Industrial - Escuela de Diseño de la Facultad de Arquitectura y Urbanismo (FAU), y cursos análogos de Ingeniería Eléctrica (quinto año), de la Facultad de Ciencias Físicas y Matemáticas (FCFM), de la misma casa de estudios. 
En este espacio creativo y exploratorio se desarrollan proyectos de mediana y alta complejidad, abordando problemáticas reales, que permiten reforzar las competencias particulares de cada especialidad, además de mejorar la metodología desarrollada para germinar y fortalecer las competencias transversales que se necesitan en la transdisciplina, como el mismo metadiseño.

Esta experiencia académica-formativa, se ha desarrollado durante seis semestres consecutivos, articulando el conocimiento y concordando continuamente las formas de abordar los problemas propios de cada disciplina. Este proceso es simbiótico, ya que requiere la permanente colaboración entre los profesores, académicos y por sobre todo de los estudiantes, siendo estos últimos agentes relevantes en la construcción de una estructura organizacional y de trabajo común donde confluyen visiones, conocimientos y experiencias radicalmente distintas pero capaces de generar un saber colectivo que da forma a productos y servicios más sustentables.

Respecto a los objetivos concretos, esta instancia está orientada a la realización de un proyecto integral de diseño con un equipo multidisciplinario, donde estudiantes de ingeniería y diseño trabajan conjuntamente, aportando su experiencia, expertise y filosofías de trabajo, para solucionar una problemática de impacto real. Los estudiantes deben comprender su rol como facilitador o posibilitadores de condiciones dentro del sistema. Para ello, la metodología se basa, en líneas generales, en conocer el contexto problema y los usuarios, proponer un espectro de soluciones, definir criterios y prototipar la idea inicial. Si bien se entiende que una solución a una problemática se puede abordar desde distintas perspectivas, teniendo una gama de resultados en distinto orden y niveles (cultura, sistemas, políticas, servicios, artefactos, entre otros), en este caso imponemos como una restricción que las soluciones deben ser sistémicas, con distintas etapas y con un primer resultado tangible. Hay dos razones que justifican esta restricción; por un lado, el resultado tangible se convierte en un punto de encuentro concreto, que materializa la simbiosis entre el diseño y la ingeniería; por otro, al ser sistémico y por etapas, permite que el desarrollo del proyecto se pueda continuar posteriormente en otros cursos superiores de otras especialidades y proyecto de titulación con otros estudiantes, quienes no necesariamente serán los mismos autores (co-creación y co-autoría).

El proyecto busca ser un espacio común donde se construyan las estructuras relacionales entre los actores y los sistemas sociales para crear nuevos espacios, abordando los quiebres, deseos y oportunidades desde las relaciones tetraedronales ${ }^{10}$. Además, se plantean ciertas competencias:

- Ética Profesional: cuestiona el actual rol del diseñador/ingeniero(a) y sus prácticas profesionales en busca de un cambio de paradigma más autopoiético y menos darwiniano, hacia un desafío más colaborativo y participativo. Reflexiona y argumenta acerca de la responsabilidad ética, social y medioambiental del futuro diseñador/ingeniero(a) para la creación de un nuevo discurso de diseño.

- Modos de desarrollo: identifica nuevos escenarios para las prácticas de diseño e ingeniería, dentro y fuera de las fronteras de la disciplina y la profesión, para reformular sistemas de producción, acumulación, patrones de consumo y políticos, entre otros. Emplea sus 
habilidades conceptuales, teóricas y prácticas para producir diseño más allá del valor de cambio, posicionándose en áreas donde el diseño no está presente aún.

- Sustentabilidad Ambiental: reconoce e integra factores medioambientales, de organización natural y de sustentabilidad social en los procesos y prácticas de diseño como claves para la co-existencia y co-creatividad. Analiza y reflexiona los impactos ecológicos y sociales en el diseño de nuevos escenarios/sistemas/servicios/productos, en base a otras áreas y con otros actores (profesionales, comunidades, organizaciones, ciudadanos, etc.).

- Inclusión Social: fomenta prácticas colaborativas en el ámbito público y privado para la co-autoría, co-creación y el co-diseño. Explora y diseña modos de organización e intercambio de recursos en función de procesos participativos, colaborativos, transdisciplinares y consensuados.

En esta instancia se enfatiza lo colectivo en lugar de lo individual, el auto-conocimiento como paso para alcanzar conocimiento (Sócrates), el trabajo en forma más relacional, holística, colaborativa, informada y responsable; "diseñar sin la idea de un producto sino como un proceso o camino de vida en sí mismo (Jones, 1980)", además se incentiva y valora el "diseño" de palabras e ideas, partiendo de la base que "escribir es importante, porque esto puede agudizar la crítica y la auto-reflexión propia del diseño" (Wood, 2007). Trabajar en un nivel de diseño que trascienda las actuales fronteras de las prácticas especializadas, de manera transdisciplinar y en equipos multidisciplinares. Debiendo dar cuenta de sistemas complejos, entornos o medioambiente (dependiendo el tema abordado), tales como transporte, currícula educacional, bienestar social, sistemas bancarios, equidad, modos de habitar, modos de producción de alimentos, sistemas de salud, sistemas energéticos, etc. Asimismo, establecer relaciones de quiebre-visión de futuro en lugar de hablar sobre problema-solución, ya que esta relación puede estar semánticamente cargada -por razones históricas y de lenguaje- de una connotación negativa y limitada, e inevitablemente nos dispone racional y emocionalmente de manera distinta, en un mundo complejo y cambiante.

Este documento busca abrir la reflexión e intercambio de ideas, metodologías y prácticas sobre los alcances de la educación en diseño, la importancia de la relación de ésta con otras disciplinas al nivel formativo, su implicancia en los actuales sistemas de consumo, y el rol como agente crítico y de cambio hacia modos de desarrollo más sustentable, para la transformación, la co-creación y la co-existencia.

\section{Notas}

1. Disponible en: http://unesdoc.unesco.org/images/0014/001486/148654so.pdf

2. Datos de Red de Pobreza Energética, Universidad de Chile (RedPE). Disponible: http:// redesvid.uchile.cl/pobreza-energetica/category/agenda/.

3. Encuesta CASEN, Observatorio Social, Ministerio del Desarrollo Social y Familia. Disponible en http://observatorio.ministeriodesarrollosocial.gob.cl/casen-multidimensional /casen/casen_2017.php. 
4. Our Common Future: Report of the World Commission on Environment and Development. Extraído el 11/10/2019 de: http://www.un-documents.net/ocf-02.htm\#I.

5. Ley No20. 290 del Marco para la Gestión de Residuos, la Responsabilidad Extendida del Productor y Fomento al Reciclaje. Disponible en: https://mma.gob.cl/wp-content/ uploads/2015/06/Ley-REP-Ley-No20920.pdf. Ley N²0.920, extraído el 11/10/2019.

6. En: Ezio Manzini: hacia un nuevo Humanismo. Por Marta Almeida | Nadia Horta | Camila Offenhenden | Publicado en diCom 2017 | Publicación original Revista iF: Diez desafíos del diseño, № 10, Buenos Aires, Centro Metropolitano de Diseño (CMD), septiembre de 2015. Extraído el 09/10/2018. https://maestriadicom.org/articulos/entrevistacon-ezio-manzini-hacia-un-nuevo-humanismo/.

7. Co-creación es usado aquí como el proceso de significado personal y social de los artefactos y actividades producto de un sistema participativo germinado a través del metadiseño (Giaccardi, 2005).

8. Disponible en: http://ingenieria.uchile.cl/facultad/ingenieria-y-ciencias-2030/113491/ proyecto.

9. Disponible en: http://ingenieria.uchile.cl/facultad/ingenieria-y-ciencias-2030/beauchef -proyecta/124183/mision-y-vision.

10. En: Wood, John (2007). Designing a discursive framework for collaboration; the tetrahedral model as a mapping tool. Apuntes del programa MA in Design Futures, Goldsmiths, University of London UK.

\section{Referencias Bibliográficas}

Dinur, B. (2007). What can metadesigners learn from ecology? Metadesign Colloquium: The idea of metadesign. Goldsmiths: University of London.

Escobar, A. (2016). Autonomía y diseño, la realización de lo comunal. Popayán: Editorial Universidad del Cauca.

Etymology. (2007). Dictionary of etymology. Recuperado el 08/09/2019 de http://www. etymonline.com.

Fuad-Luke, A. (2009). Design Activism: Beautiful strangeness for a sustainable world. London: Routledge.

Giaccardi, E. (2003). Principles of metadesign: processes and levels of co-creation in the new design space. (A Thesis submitted to the University of Plymouth for the degree of Doctor of Philosophy). United Kingdom: University of Plymouth.

Giaccardi, E. (2005a, 10/11/2006). Metadesign as an emergent design culture. MIT Press Journal. Leonardo 38, No4. Disponible en: https://www.mitpressjournals.org/doi/abs/10 $.1162 / 0024094054762098$ ?journalCo $=\&$ mobileUi $=0$

Giaccardi, E. (2005b). Sowing the seeds for co-creation: in the multidimensional ppace of metadesign. Attainable Utopias. Disponible en: http://www.attainable-utopias.org/tiki/ Co-Creating-a-MultiDimensional-Metadesign.

Jones, J C. (1980). Design methods: seeds of human futures. New York: John Willey \& Sons Ltda. 
Luengo, E. (2012). Una propuesta conceptual. La disciplina y sus desafíos en la universidad. Interdisciplina y transdisciplina: aportes desde la investigación y la intervención social universitaria (Pp. 09-24). Guadalajara: ITESO Universidad Jesuita de Guadalajara.

Manzini, E. (2007). Designing networks and metadesign. Metadesign Colloquium: The idea of metadesign, Goldsmiths, University of London.

Maturana, H. \& Varela, F. (1987). The tree of knowledge: The Biological Roots of Human Understanding. Boston \& London: Shambhala Publications.

Maturana, H. (1997). Metadesign. Chile: Instituto de Terapia Cognitiva (INTECO). Recuperado el 10/12/2006.

Papanek, V. (1985). Design for the real world: human ecology and social change. London: Thames \& Hudson.

Wood, J. (2005). The tetrahedron can encourage designers to formalise more responsible strategies. Journal of Art, Design \& Communication. Vol 3. Issue 3. United Kingdom: Linda Drew

Wood, J. (2007d). A Method for Co-Designers: Mapping Ethical Relations in Metadesign. London: Paper given on the MA Design Futures Programme at Goldsmiths, University of London: 10.

Wood, J. (2007). Design for Micro-Utopias: Making the Unthinkable Possible. England: Gower Pub Co.

\begin{abstract}
In the current, complex and changing global scenario, the sense of urgency in achieving carbon neutrality, reducing the effects of greenhouse gases and the effects of climate change, are part of the challenges we face, not only because it affects the dependence of human beings of fossil fuels but also because of the way we live, how we organize ourselves as a society and their respective economies. Naomi Klein (2014), expresses it clearly when he says: this changes everything. For science, technology and design, innovation and sustainability are fundamental in the development of a new model, approaches and integral proposals, which provide solutions at different levels and dimensions to implement the structural transformations that the planet and society as a whole you need.
\end{abstract}

Keywords: Sustainability - Metadesign - Transdiscipline - Transformation - Climate change.

Resumo: No cenário global atual, complexo e em mudança, o senso de urgência em alcançar a neutralidade do carbono, reduzindo os efeitos dos gases de efeito estufa e os efeitos das mudanças climáticas, fazem parte dos desafios que enfrentamos, não apenas porque afeta a dependência de seres humanos a partir de combustíveis fósseis, mas também por causa da maneira como vivemos, como nos organizamos como sociedade e suas respectivas economias. Naomi Klein (2014) expressa isso claramente quando diz: isso muda tudo. Para ciência, tecnologia e design, inovação e sustentabilidade são fundamentais no desenvolvimento de um novo modelo, abordagens e propostas integrais, que fornecem soluções 
em diferentes níveis e dimensões para implementar as transformações estruturais que o planeta e a sociedade como um todo você precisa.

Palavras chave: Sustentabilidade - Metadiseño - Transdisciplina - Transformação - Mudança climática. 